\title{
Article \\ Personality Traits Modulate the Effect of tDCS on Reading Speed of Social Sentences
}

\author{
Cristian Reyes ${ }^{1, *}$, Iván Padrón ${ }^{2,3}$ (D) Sara Nila Yagual ${ }^{4}$ and Hipólito Marrero ${ }^{2,5}$ (D) \\ 1 Experimental Psychology Lab, Department of Psychology, Carl von Ossietzky University of Oldenburg, \\ 26129 Oldenburg, Germany \\ 2 Instituto Universitario de Neurociencia, Universidad de La Laguna, 38200 San Cristóbal de La Laguna, Spain; \\ ivpadron@ull.edu.es (I.P.); hmarrero@ull.es (H.M.) \\ 3 Departamento de Psicología Evolutiva y de la Educación, Universidad de La Laguna, \\ 38200 San Cristóbal de La Laguna, Spain \\ 4 Facultad de Ciencias Sociales y de la Salud, Universidad Estatal Península de Santa Elena, \\ La Libertad 241702, Ecuador; pssarukita@hotmail.com \\ 5 Departamento de Psicología Cognitiva, Social y Organizacional, Universidad de La Laguna, \\ 38200 San Cristóbal de La Laguna, Spain \\ * Correspondence: cristian.reyes.moreno@uni-oldenburg.de
}

check for updates

Citation: Reyes, C.; Padrón, I.; Nila Yagual, S.; Marrero, H. Personality Traits Modulate the Effect of tDCS on Reading Speed of Social Sentences.

Brain Sci. 2021, 11, 1464.

https://doi.org/10.3390/

brainsci11111464

Academic Editor: Paola Marangolo

Received: 3 October 2021

Accepted: 2 November 2021

Published: 5 November 2021

Publisher's Note: MDPI stays neutral with regard to jurisdictional claims in published maps and institutional affiliations.

Copyright: (c) 2021 by the authors. Licensee MDPI, Basel, Switzerland. This article is an open access article distributed under the terms and conditions of the Creative Commons Attribution (CC BY) license (https:/ / creativecommons.org/licenses/by/ $4.0 /)$.

\begin{abstract}
In this case, 62 university students participated in the study, in which a between-subjects design was adopted. Participants were also given the behavioral approach system (BAS) and behavioral inhibition system (BIS) scales. Participants had to read a list of 60 sentences with interpersonal and neutral content: 20 approach ("Pedro accepted Rosa in Whatsapp"), 20 avoidance ("Pedro Blocked Rosa in Whatsapp") and 20 neutral ("Marta thought about the causes of the problem"). After reading them, they were subjected to 20 min of transcranial direct current stimulation (tDCS) in one of the two conditions: anodal (31) or sham (31). After tDCS, they had to read other list of 60 sentences matched in approach, avoidance and neutral contents with the former list. We found significant improvement in reading speed after anodal stimulation for social and neutral sentences. Regarding affective traits, we found that anodal stimulation benefitted reading speed in low-BIS and low-BAS participants and had no effect in either high BAS or high BIS participants. In addition, tDCS improvement in reading speed was significantly lower in avoidance sentences in low-BIS (avoidance) participants. We discuss these results at the light of previous research and highlight the importance of approach and avoidance traits as moderators of tDCS effects.
\end{abstract}

Keywords: approach/avoidance intentionality; relationship action-sentences; tDCS; reading; superior temporal sulcus

\section{Introduction}

Intentionality is a basic component of understanding the minds and behaviors of others. In this regard, the temporal lobe (anterior temporal lobe, superior temporal sulcus, middle and superior temporal gyrus) and the precuneus and temporo-parietal junction constitute a "mentalizing" network [1-3] that encodes intentionality. It is relevant to distinguish between representation of intentions as mental states not associated with current actions, and representation of intentions and goals that are inherent in perceived actions. The latter involves a neural system particularly associated with the Superior Temporal Sulcus (STS) and is recruited for action understanding [4]. Approach and avoidance intentionality are at the serve of regulating adaptive conduct. Other pieces of research have examined relevant aspects of cognition and adaptive behavior, as its relationship with affective stimuli processing. For example, it has been suggested that medio-frontal negativity, a component of the event-related brain potential generated in ACC/mPFC, tracks the timing of salient events and reports an error signal for outcomes occurring at unexpected times [5]. Similarly, results of [6] showed how the ventromedial prefrontal cortex (vmPFC) 
is involved in the acquisition of fear conditioning (i.e., learning), as it is fundamental for the evaluation and representation of action's value needed to produce sustained conditioned physiological responses. Furthermore, findings from [7] confirmed the modulation effect that emotional stimuli have on executive control, since they interfere with inhibitory control of behavior, given situational demands. Moreover, activation of this mentalizing network to process social information is usually stronger in the right hemisphere $[8,9]$.

Previous research has highlighted how the interpretation of social signs is essential in guiding appropriate behaviors. Solid psychological evidence suggests that proximity of fear stimuli (angry faces) evoked redirection of attention. This attention redirection would be at the serve of enhancing the defensive function of the so-called Peripersonal Space (PPS) [10]. Similarly, results from a recent study [11] show that interpretation of potentially threatening situations, such as others' proximity, triggers a number of physiological responses that help to regulate the distance between ourselves and others during social interaction. Within the mentalizing network, the STS and brain areas around it have been shown to be particularly involved in processing communicative intention for interactions by means of gaze (direct vs. averted) in social perception [12-16] and mutual liking [17]. It has also been shown that approach intentionality causes greater activation of posterior right Superior Temporal Sulcus (rSTS) than avoidance. In a fMRI study [18], brain activation in response to a stranger initiating or avoiding social interaction was measured. Participants viewed an animated character approaching down a virtual hallway, who shifted his gaze either toward or away from the participant. Mutual gaze (approach) caused a greater activation in this brain region than averted gaze (avoidance). These studies usually focused on demonstrating that STS is responsible for action intentionality and social contexts, and not just for the more physical aspects of actions $[12,13]$. However, it could be that the STS is a brain area specifically recruited for processing intentionality for relationships.

Whereas social perception of approach/avoidance intentionality activates posterior aspects of rSTS, several studies have supported that more abstract and conceptual processing of relationship intentionality recruits more anterior to middle aspects of rSTS. For example [19] (see also [20]), using a version of the Heider and Simmel animation task in a fMRI study, reported activation of more anterior aspects of rSTS when participants judged "friendship" from simple geometric shape interactions. Similarly [4] have reported activation along the full length of rSTS when participants observed Heider and Simmel animations and made social intentional judgements of interactions.

Beyond action observation, language describes how individuals interact with other people by means of social actions that conceptually involve approach ("pro-stimulus") and avoidance ("against-stimulus") intentionality [21,22]. For example, "Alejandro accepted/rejected Marta in his group". Approach and avoidance would constitute a semantic frame or category to be systematically encoded for understanding this type of actions, which shows an individual's intentional direction towards other people, and has an adaptive role. Thus, if approach/avoidance gives meaning to relationship actions, we could expect a greater activation in more anterior aspects of STS to process them in social relationship actions.

\subsection{Non-invasive Brain Stimulation (NIBS)}

Non-invasive brain stimulation (NIBS) techniques are widely used to modulate activity of brain areas. Within these techniques, transcranial electrical stimulation (tES) and transcranial magnetic stimulation (TMS) are two of the most known types of NIBS that modulate neural activity in a different way each [23]. In case of TMS, a short electromagnetic current is generated in the brain, which derive in supra-threshold activations in neurons. However, tES does not generate action potential in neurons but modulate neurons' activity by producing sub-threshold modulations of membrane potentials [24]. In case of tDCS, direct electrical current is applied through scalp, penetrating skull and modulating cortical excitability [25]. Duration of these effects depend on parameters of 
stimulation, such as current density, stimulation duration and/or geometrical montage of electrodes, as well as direction of effects strongly depends on polarity [26].

Hence, selectively changing neuronal activity may be linked with modulation in cognitive functions. An example is an experiment of [27], who demonstrated that NIBS delivered on the prefrontal cortex may disrupt physiological response associated with fear memories. They run an experiment in which participants were conditioned to pictures of different indoor scenes by applying an electrical mild shock to the left inner wrist. On the next day ( $24 \mathrm{~h}$ after acquisition), reactivation of the association learned was carried out by showing the conditioned image with no electrical shock, but repetitive TMS was applied $10 \mathrm{~min}$ after the reactivation. In 2 out of 5 groups where reactivation occurred, rTMS was applied either on the right or left dorsolateral prefrontal cortex (r-dlPFC, 1-dlPFC). On a third day ( $48 \mathrm{~h}$ after acquisition), there was a recall test where there was no association between the electrical shock and the conditioned images. For all the three phases, skin conductance response (SCR) was recorded. Statistical analysis showed that differences in SCR between signaled and non-signaled conditioned stimuli were significantly decreased during the recall test due to rTMS administration on both, right and left dlPFC, in comparison to other groups with different rTMS location, sham or recall test assessed on the second day.

However, the application of NIBS is not only on normal, but also in clinical population. For example [28], applied tDCS in 30 schizophrenic patients. Half participants received sham stimulation and the other half received tDCS at $2 \mathrm{~mA}$ for $20 \mathrm{~min}$ to see the effects of tDCS on auditory hallucinations. The electrodes were placed according to $10 / 20$ system. Anode was placed at a point in between F3 and FP1, targeting dorsolateral prefrontal cortex, and the cathode was placed at point midway T3 and P3, targeting left temporoparietal junction. They found a significant reduction of auditory hallucinations in the tDCS group 5 days, 1 month and even 3 months after tDCS treatment. Moreover, they found a significant reduction in negative dimension of schizophrenia symptomatology according to PANSS (Positive and Negative Syndrome Scale) was found 5 days after the treatment sessions. Other neuropsychiatric disease in which the use of NIBS has been researched is depression (see [29]. For instance [30], found an improvement in mood in depression patients in scores of the MADRS (Montgomery-Asberg Depression Rating Scale) by comparing those patients assigned to a sham-tDCS group with those patients in an actual tDCS group. They placed the anode on the left dorsolateral prefrontal cortex (which was identified as fP3) and the cathode was placed over the lateral parts of the contralateral orbit, which was localized at F8 position accordingly to the $10 / 20$ system.

Regarding tDCS effects on the STS and relationship-actions processing, it has been found [31] found a greater improvement in discriminability of approach sentences in a memorization task after applying anodal tDCS on the rSTS, in comparison to either sham or cathodal stimulation [31]. However, the question whether the advantage of approach content could start before memorization, such as during sentence reading remains open. Likewise, in this study [31], only approach vs. avoidance sentences were tested without including neutral sentences.

In the field of reading processes, tDCS has been applied and led to significant improvement in different reading subprocesses in both normal readers and readers with dyslexia (see [32]). In these studies, a typical target area is the left temporo-parietal cortex. With regards to reading efficiency, it has been evaluated whether reading efficiency could be improved by applying tDCS on the temporo-parietal junction on both hemispheres, localized at CP5 or CP6, according to the 10-20 EEG system [33]. It was found that anodal stimulation caused a greater effect than cathodal stimulation in both reading efficiency and speed for single words.

As can be seen, effects of tDCS on reading efficiency have been examined in left temporo-parietal areas. Language is dependent on left hemisphere activity. By contrast, right hemisphere is specialized in social processing, and the temporal area around the STS is in processing social intentionality [8,9]. In accordance with the Embodied Simulation Theory $[34,35]$, understanding social relationship action-sentences would involve expe- 
riential simulation of approach/avoidance intentionality. For that reason, we examine for the first time the improvement of reading speed in sentences with social contents by stimulating the temporal area in the right hemisphere.

\subsection{Moderation of tDCS Effect by Approach and Avoidance Personality Traits}

We also explored whether an effect of tDCS on reading speed could be moderated by approach and avoidance traits (behavioral approach system (BAS) and behavioral inhibition system (BIS) [36]. Previous research has found that only low approach trait participants show improvement following tDCS [37-39].

We consider that the moderator effect of trait could be exerted by affecting attention allocation in possibly two different ways. One way is by a motivational bias. In this case, we expected that greater cognitive resources furnished by tDCS would be used more on processing approach sentences than avoidance by high BAS participants, whereas high BIS participants would use more resources processing avoidance sentences. Thus, we predict greater $\mathrm{tDCS}$ effect on reading speed in approach than in avoidance sentences for high BAS trait, and in avoidance than in approach sentences in high BIS trait. The other way is related to a deficit in attention allocation. Previous research has suggested that high approach trait (impulsivity) is associated with less concentration, more distractibility, and less attentional narrowed focus on a given task $[38,40,41]$. In the case of avoidance trait, previous research has clearly showed that fearfulness and anxiety disturb the capacity for allocation of attentional resources to a particular task (see [42]). Thus, we would expect high-BAS and high-BIS traits participants to be less able to take advantage of additional processing resources plausibly furnished by anodal tDCS for the reading task. Thus, we predict a poorer reading improvement both in approach and avoidance sentences in high BAS and BIS compared to low BAS and BIS participants.

\section{Materials and Methods}

\subsection{Design}

A $3 \times 2$ factorial design was used, with Direction (approach vs. avoidance vs. neutral) as within-subjects factors and Stimulation (anodal vs. sham) as between-subjects factor. The dependent variable was improvement in reading times that was measured as the difference in reading times before and after tDCS.

\subsection{Participants}

In this case, 62 undergraduate students ( 54 females, $M=19.95, S D=2.33$ ) from the University of La Laguna (La Laguna, Spain) participated in the experiment in exchange for course credits. The minimal sample size to generate appropriate statistical power (80) with 0.05 alpha bilateral for a small effect size $\left(\eta^{2}=0.026\right)$ for two independent means (anodal group vs. sham) was established at $N=31$ for each group (see [43]). Inclusion criteria included: being right-handed according to the Edinburgh Handedness Inventory [44]. Exclusion criteria were suffering from epilepsy (or having close relatives affected), migraine, brain damage, cardiac, neurological or psychiatric disease, having any injury or subcutaneous metal in any of the two parts where electrodes would be set. In this study, 31 participants were subjected to the anodal condition and 31 to the sham condition.

\subsection{Stimuli}

We selected a pool of 120 sentences from Marrero et al. [21], 80 approach and avoidance sentences that had been controlled for linguistic factors such as sentence length and number of syllables, and psycholinguistic factors such as their imaginability, and 40 neutral sentences with no social content. Table 1 showed examples of sentences in the different conditions. Among these sentences, $60 \%$ of proper names were female and $40 \%$ were male names. There is greater percentage of female students in Psychology degree. Thus we could expect more everyday female interactions which we try to translate into sentences. 
Table 1. Examples of approach, avoidance and neutral sentences with questions.

\begin{tabular}{|c|c|c|c|}
\hline Sentence & Direction & Question Example & Correct Answer \\
\hline $\begin{array}{c}\text { Pedro/aceptó a } \\
\text { Rosa/en Whatshapp } \\
\text { (Pedro/accepted } \\
\text { Rosa/in Whatsapp) }\end{array}$ & Approach & $\begin{array}{c}\text { ¿Dice que Pedro } \\
\text { aceptó a Rosa en } \\
\text { Whatshapp? } \\
\text { (Is it stated that Pedro } \\
\text { accepted Rosa in } \\
\text { Whatshapp?) }\end{array}$ & Yes \\
\hline $\begin{array}{l}\text { Pedro/bloqueó a } \\
\text { Rosa/en Whatshapp } \\
\text { (Pedro/blocked } \\
\text { Rosa/in Whatshapp }\end{array}$ & Avoidance & $\begin{array}{l}\text { ¿Dice que Pedro } \\
\text { aceptó a Rosa en } \\
\text { Whatshapp? (Is it } \\
\text { stated that Pedro } \\
\text { accepted Rosa in } \\
\text { Whatshapp?) }\end{array}$ & No \\
\hline $\begin{array}{l}\text { Verónica/dedujo el } \\
\text { precio/del abrigo } \\
\text { (Verónica/deduced } \\
\text { the price/of the coat) }\end{array}$ & Neutral & $\begin{array}{l}\text { ¿Dice que Verónica } \\
\text { dedujo el precio del } \\
\text { abrigo? (Is it stated } \\
\text { that Verónica } \\
\text { deduced the price of } \\
\text { the coat?) }\end{array}$ & Yes \\
\hline
\end{tabular}

\subsection{Affective Tests}

The behavioral inhibition system (BIS) and behavioral activation system (BAS) scales were measured by the Carver and White scales [45]. BAS measures individual sensitivity to reward, and BIS sensitivity to punishment [36]. Both BAS and BIS scales were reliable in this study: $\alpha=0.851$ and $\alpha=0.825$, respectively.

\subsection{Procedure}

\subsubsection{Experimental Task}

The approach and avoidance content of the pool of 120 sentences from Marrero et al. [21] was counterbalanced in two lists. If a sentence is approach in one list, then it appeared as avoidance in the other list, and vice versa. Then we split each list into two lists of 60 sentences each (20 sentences for each type of sentence: approach, avoidance, and neutral), one to be passed before tDCS stimulation, and the other after stimulation inasmuch we were interested in measuring reading improvement after tDCS. The order of the lists was counterbalanced as follows: list 1-tDCS-list 2; list 2-tDCS-list 1; list 3-tDCS-list 4; list 4-tDCS-list 3. Participants were randomly assigned to each sequence order, taking into account that they were all from the first course of Psychology degree, and thus are assumed to be homogeneous in cognitive reading skills. Sentences were randomly presented to the participants in each of the counterbalanced sets. Participants were told that the task consisted of reading segmented sentences one by one displayed on a computer screen for comprehension, while they were seating in front of it.

At the start of the experiment, participants were given seven sentences to practice. Then, they were given 60 sentences, 20 for each experimental within-subject condition: Approach vs. Avoidance vs. Neutral. Each sentence presentation started with a cross point displayed in the middle of the screen for $750 \mathrm{~ms}$. After an interval of $150 \mathrm{~ms}$, one sentence was displayed. Sentences presentation was segmented (three segments, see Table 1); for example, "Pedro/bloqueó a Rosa/en el Whatsapp" ("Pedro/blocked Rosa/in Whatsapp."). Each segment was displayed till the participant pressed the corresponding button. After $750 \mathrm{~ms}$ a new sentence appeared. To avoid participants' superficial reading, 36 sentences were immediately followed by a question on the contents just read (e.g., "Is it stated that Pedro blocked Rosa?"). This question had either a positive or a negative response half the times and remained on the screen for $5000 \mathrm{~ms}$ or until a response was made. Feedback on correctness and time required was given to the participants and displayed for $2000 \mathrm{~ms}$ These questions were aimed at keeping the attention of participants 
on reading comprehension. After a delay of $750 \mathrm{~ms}$, a new sentence was displayed. Response recordings and stimuli presentation were controlled by E-Prime 2.0 software (Psychology Software Tools, Pittsburgh, PA, USA).

\subsubsection{Protocol for $\mathrm{tDCS}$ Application}

A CE-certified battery-powered stimulator (neuroConn DCSTIMULATOR. neuroConn $\mathrm{GmbH}$, Albert-Einstein-Str. 3, 98693 Ilmenau, Germany) was used for the non-invasive tDCS current conduction with an intensity of $2 \mathrm{~mA}$. The electrodes of the equipment used were rubber, with one being $5 \times 5 \mathrm{~cm}$ and $7 \times 5 \mathrm{~cm}$ the other. Both were covered with sponges soaked in saline to transfer direct current, which resulted in a density of $0.08 \mathrm{~mA} / \mathrm{cm}^{2}$ and $0.057 \mathrm{~mA} / \mathrm{cm}^{2}$, respectively. The smaller electrode was placed on the scalp in accordance with International System 10-20. The selected area was T8, as it is the most appropriate for the stimulation of the temporal region of interest. The other electrode was extracranially placed on the contralateral shoulder to minimize its effects on the brain and have higher focality at the region of interest [46]. We stimulated BA 22 and BA 21 brain areas overlapping medial aspects of rSTS, as shown in Figure 1. In addition, the stimulated area is a part of the so-called mentalizing network [2], specialized in processing social intentionality.
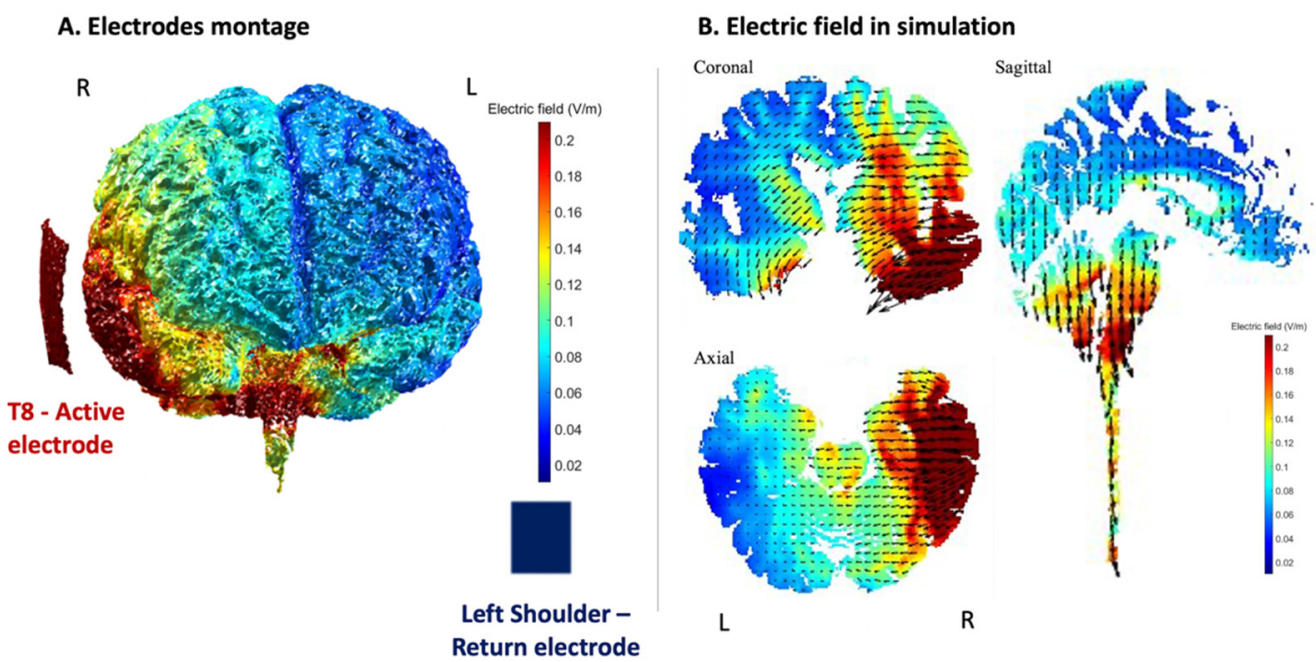

Figure 1. Computational representation of the electric field intensity generated by our transcranial direct current stimulation (tDCS) montage with reference to anode (T8) and an extracephalic cathode. Units are in $\mathrm{V} / \mathrm{m}$. The simulation was run using ROAST (realistic volumetric approach to simulate transcranial electric stimulation) [47]. (A) Electrode montage and, (B) Electric field in simulation in three slides (axial, coronal and sagittal planes).

The stimulation application time was $20 \mathrm{~min}$ plus a Fade in and Fade out of $15 \mathrm{~s}$ both. The stimulation time was established based on previous studies of tDCS (e.g., [48,49]). During the false tDCS (sham) condition, the constant current only lasted 45 s: Fade in: 15 s, $15 \mathrm{~s}$ maximum intensity and Fade out: $15 \mathrm{~s}$.

\subsection{3. tDCS Procedure}

Upon arrival at the laboratory, participants were informed about the general aim of the study. They all filled in a personal data form and a questionnaire to screen for exclusion conditions and signed an informed consent form. Participants were told that the objective of the study was to examine the effect of brain stimulation on cognitive enhancement. They were not informed about the tDCS condition they had been assigned to. Thus, in both $\mathrm{tDCS}$ conditions, they were supposed to believe that they were being positively stimulated. No direct assessment of blinding assessment was performed. None of them reported suffering from epilepsy (nor having close relatives affected), migraines, brain damage, cardiac disease, or other psychological or medical conditions. All participants 
were right-handed, according to the Edinburgh Handedness Inventory [44]. The ethical committee of the University of La Laguna approved the study: (CEIBA 2017-0272).

Participants were also given the BIS/BAS scales. Subsequently, they performed the first set of sentences of the experimental task. After that, electrodes were placed, and tDCS stimulation started in accordance with the tDCS protocol. Immediately after removing the tDCS equipment, participants performed the second set of sentences. Once this task was finished, they were thanked for their cooperation, and a short explanation of the experimental procedure was given to them for debriefing. Likewise, they were advised not to discuss the experiment with other potential participants.

The experimental session lasted approximately $55 \mathrm{~min}$. The stimulation parameters were considered safe [50]. We asked participants to inform us of any adverse event during tDCS application. We asked the subjects again at the end of the experimental session and told them to let us know whether they felt such effects in the following days. Some volunteers informed us of mild and transient adverse effects (see [51]) during intervention. Table 2 shows the type of adverse effect, the severity of the effect and the percentage of the participants that experienced them.

Table 2. Adverse effects, severity, and percentage of participants that experienced them in the tDCS study.

\begin{tabular}{ccc}
\hline Type of Effect & Severity & Percentage \\
\hline Tingling & Mild & $27.14 \%$ \\
\hline Itching & Mild & $67.14 \%$ \\
\hline Warm & Mild & $4.28 \%$ \\
\hline
\end{tabular}

\section{Results}

Sentence reading times above/under $2.5 \mathrm{SD}$ of the participant mean (1.8\%) were removed from the analysis. Two participants were removed from the analysis as they exceeded the criteria of less than $25 \%$ of incorrect responses to the questions.

We assumed a normal distribution of improvement in reading speed. The Saphiro-Wilk test supported a normal distribution of improvement $(p>0.05)$. We carried out an ANOVA with Stimulation (anodal vs. sham) as a between-subjects factor and Direction (approach, avoidance and neutral) as a within-subjects factor. We used the latency to question in neutral sentences after tDCS as a covariate to further control attentional variability in the reading task. Descriptive data of reading improvement are shown in Table 3. Likewise, in Figure 2, the score distributions for tDCS conditions in each type of sentence are shown.

Table 3. Descriptive statistics of reading improvement as a function of the type of sentence and the tDCS conditions.

\begin{tabular}{ccccc}
\hline Direction & Stimulation & Mean & SD & N \\
\hline Approach & Anodal & 425.81 & 465.06 & 31 \\
\hline Avoid. & Sham & 288.82 & 280.42 & 29 \\
\hline \multirow{2}{*}{ Neutral } & Anodal & 284.83 & 327.73 & 31 \\
\hline & Sham & 171.56 & 170.30 & 29 \\
\hline & Anodal & 363.5 & 418.53 & 31 \\
\hline
\end{tabular}




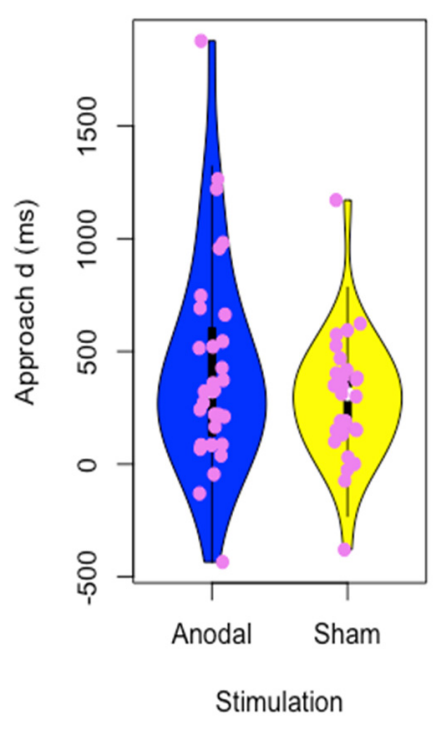

(a)

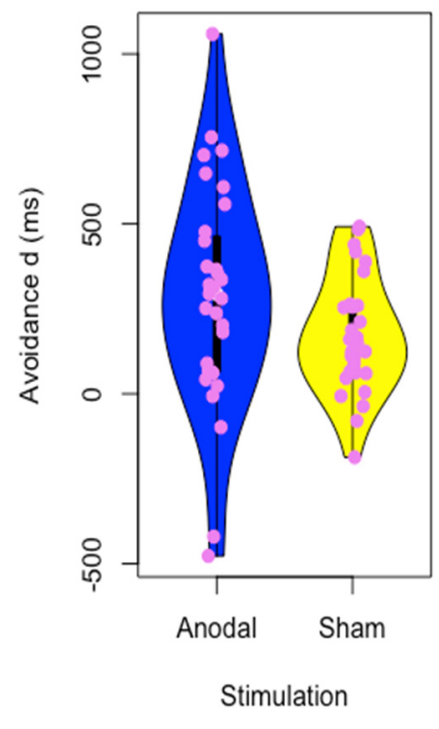

(b)

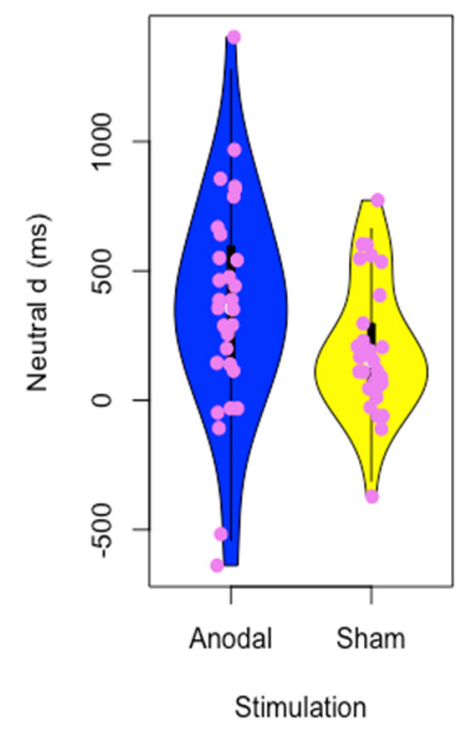

(c)

Figure 2. Distribution of speed reading improvement for tDCS conditions in the three types of sentences: approach (a), avoidance (b) and neutral (c).

The main effect of Stimulation was significant, $\left.\mathrm{F}(1,58)=4.174, p<0.046, \eta p^{2}=0.068\right)$. Anodal stimulation improved reading speed for all the types of sentences in contrast to sham condition (see Table 3). The main effect of Direction was marginally significant, $\mathrm{F}(2,59)=2.896, p=0.064, \eta p^{2}=0.094$. Reading improvement was greater for approach than for avoidance sentences $\left(\mathrm{M}_{\text {Diff. }}=129.521, S D=225.479\right), t(57)=4.45, p<0.001$; improvement was also greater for approach than for neutral sentences $\left(M_{\text {Diff. }}=76.823\right.$, $S D=318.953$ ), marginally significant, $t(57)=1.866, p=0.087$; and for neutral than for avoidance sentences $\left(M_{\text {Diff. }}=52.697, S D=223.675\right)$, marginally significant, $t(57)=1.82$, $p=0.073$. The interaction Direction $\times$ Stimulation was not significant, $p>0.10$.

\subsection{Moderation of tDCS by Affective Traits}

We examined modulation by affective traits of tDCS effect on reading improvement. Modulatory analyses are aimed at examining whether $\mathrm{tDCS}$ affected reading performance of participants depending on having a higher or lower levels either in BAS or in BIS traits. Participants were classified in BAS trait as 'low' (those who scored below the 35th percentile score of 1.70), 'medium' (between the 35th and 65th percentile), and 'high' (higher than the 65th percentile score of 2.14) taking into account the whole sample, and the range was 1.15-3.38. Likewise, they were classified in BIS trait as 'low' (those who scored below the 35th percentile score of 2.01), 'medium' (between the 35th and 65th percentile), and 'high' (higher than the 65th percentile score of 2.42) taking into account the whole sample, and the range was 1.43-3.29. We were interested in looking for differences between the low-high trait participants, therefore, intermediate levels of each trait were not of interest.

\subsection{Behavioral Approach System (BAS)}

Saphiro-Wilk test supported a normal distribution of $\mathrm{d}$ scores in participants for both Stimulation conditions $(p>0.05)$ and the two BAS groups. Following the same design as described above, we carried out two $2 \times 3$ ANOVAs on reading improvement. In the case of low-BAS participants, a main effect of Stimulation was found, $\mathrm{F}(1,19)=6.53, p=0.02$, $\eta p^{2}=0.205$. As can be seen in Table 4, anodal stimulation furnished greater improvement than sham condition in the three types of sentences. Main effect of Direction and the interaction Direction $\times$ Stimulation were not significant $(p>0.5)$. 
Table 4. Descriptive statistics of $d$ for low-BAS participants in each condition.

\begin{tabular}{ccccc}
\hline Direction & Stimulation & Mean & SD & N \\
\hline Approach & Anodal & 490.37 & 412.64 & 11 \\
\hline & Sham & 206.099 & 309.16 & 10 \\
\hline Avoid. & Anodal & 334.41 & 217.85 & 11 \\
\hline Neutral & Sham & 87.13 & 166.84 & 10 \\
\hline & Anodal & 411.12 & 420.50 & 11 \\
\hline & Sham & 81.03 & 228.46 & 10 \\
\hline
\end{tabular}

By contrast, no main effect of Direction, Stimulation or the interaction Direction $\times$ Stimulation was found in the case of high-BAS participants (Anodal: 11; Sham: 10), $p>0.10$.

\subsection{Behavioral Inhibition System (BIS)}

The same procedure as for the BAS trait was applied. Saphiro-Wilk test supported a normal distribution of reading improvement scores in participants for both Stimulationconditions $(p>0.05)$ and the two BIS groups. In the case of low-BIS participants, a main effect of Stimulation was found, $\mathrm{F}(1,19)=8.502, p=0.009, \eta p^{2}=0.321$. As can be seen in Table 5, anodal stimulation furnished a greater improvement than sham condition in the three types of sentences. Likewise, the interaction Direction $\times$ Stimulation resulted marginally significant, $\mathrm{F}(2,19)=3.181, p=0.067, \eta p^{2}=0.272$. Follow-up comparisons showed that in anodal condition reading improvement was less in avoidance sentences than in approach sentences, $t(10)=2.92, p=0.015$; and less in avoidance sentences than in neutral sentences, $t(10)=2.575, p=0.029$. By contrast, there were no significant differences in the sham condition. The main effect of Direction was not significant, $p=0.174$.

Table 5. Descriptive statistics of $d$ for low-BIS participants in each condition.

\begin{tabular}{ccccc}
\hline Direction & Stimulation & Mean & $S D$ & N \\
\hline Approach & Anodal & 583.70 & 412.52 & 11 \\
\hline & Sham & 280.76 & 417.18 & 10 \\
\hline Avoid. & Anodal & 366.82 & 263.08 & 11 \\
\hline Neutral & Sham & 169.39 & 188.28 & 10 \\
\hline & Anodal & 562.93 & 418.99 & 11 \\
\hline & Sham & 130.92 & 280.96 & 10 \\
\hline
\end{tabular}

In the case of High-BIS participants (Anodal: 11, Sham: 11), the main effect of Direction resulted marginally significant: $\mathrm{F}(2,21)=3.077, p=0.071, \eta p^{2}=0.255$. Reading improvement tended to be greater in approach sentences $(M=265,03, S D=256.19)$ than in avoidance sentences $(M=202.61, S D=247.538)$ or in neutral sentences $(M=240.10, S D=311.96)$. Neither the main effect of Stimulation nor the interaction Direction $\times$ Stimulation resulted significant.

\section{Discussion}

We examined the effect of anodal tDCS on reading improvement of social relationship sentences, both of approach and avoidance, and those without interpersonal content (neutral sentences). We found an effect of training with greater improvement for approach than for avoidance or neutral sentences. This effect was not affected by tDCS. Thus, this effect plausibly shows a certain facility to process approach and so, faster reading improvement in approach sentences from training in contrast to avoidance ones. In this regard, previous research [21] have found that avoidance sentences are judged as emotionally negative and more arousing than approach ones. In the case of words, more negative emotionality 
(see [52]) has been associated with a greater effort in reading. Importantly, we found a main effect of tDCS. Anodal stimulation improved reading speed in the three types of sentences in contrast to sham condition: as mentioned, tDCS effect was not modulated by the type of sentence.

The aim of our study has been to examine whether the advantage of approach content in the tDCS effect on sentence memorization [31] starts before, during sentence reading. Contrary to our expectations, reading speed of approach sentences did not benefit from anodal stimulation in rSTS. One plausible explanation is that the positive effect of anodal stimulation on memorization of approach content occurs after reading, during the process of encoding the meaning of the sentences. In this case, anodal stimulation would benefit cognitive accessibility of approach for better memorization. This hypothesis could be examined by using an immediate recognition task. Recognition tasks are suitable to examine content accessibility after reading (see [53]). Further research is thus necessary to study whether anodal tDCS on rSTS benefits memorization of approach sentences by enhancing the availability of approach content after reading.

As mentioned, anodal stimulation in rSTS had a general effect on reading improvement. That is, $\mathrm{tDCS}$ seems to furnish cognitive resources to reading for the three types of content: interpersonal (approach and avoidance) and neutral content. One possible explanation is that stimulation facilitated semantic processing in general, and it does not depend on the content. However, we must consider that we stimulated the right temporal area that is specialized in processing social content. Thus, we consider plausible that the three types of sentences share social content, as neutral sentences although they are not interpersonal, referring to persons. In this regard [9], have shown that more anterior and superior aspects of the right temporal area is recruited for sentence processing and person content. Thus, stimulation of medial to anterior aspects of rSTS would have an effect on reading the three types of sentences.

\section{1. tDCS Effect on Reading Speed Improvement Is Modulated by Approach/Avoidance Trait}

In terms of Approach-BAS modulated tDCS effect on reading speed, low-BAS participants showed a significant effect of anodal stimulation in reading improvement in comparison to Sham condition participants. By contrast, high BAS participants did not show any effect of $\mathrm{tDCS}$ on reading improvement. This result is in accordance with previous research that found a greater effect of anodal stimulation in low-approach (BAS) participants [37] and supports the attentional explanation, although rules out the motivational one. High approach (reward sensitivity) has been associated with less concentration, more distractibility, and less attentional narrowed focus on a given task $[38,40,41]$. Thus, high BAS participants would be less able to take advantage of additional processing resources plausibly furnished by anodal tDCS to read sentences, compared to low-approach ones.

Avoidance (BIS) trait also modulated the tDCS effect. Low-BIS participants showed a significant effect of anodal stimulation in reading improvement in comparison to sham participants. By contrast, high-BIS participants showed no effect of tDCS on reading improvement. This result also supports our attentional hypothesis. Fearfulness and anxiety would disturb the capacity for allocation of additional processing resources furnished by anodal stimulation to the task (see [40]).

Interestingly, we found that the effect of $\mathrm{tDCS}$ on reading improvement in low-BIS participants was modulated by the type of sentence. Post-hoc comparisons showed that anodal tDCS is associated with lesser improvement in avoidance sentences. This suggests a motivational bias but in the opposite direction of our motivational hypothesis. That is, participant with low-BIS (fear and anxiety) seem to be less benefited in reading speed of avoidance sentence from anodal stimulation. One plausible reason is a motivational bias: low-BIS participants paid less attention to avoidance content, and so took less advantage of cognitive resources furnished by anodal stimulation to increase their reading speed in the task. 


\subsection{Limitations}

A limitation of our study is the lack of focality of the applied stimulation. For the anatomical localization of the STS, we considered the position of electrode T8 of the EEG montage; however, aspects such as the anatomical variability across subjects and the lack of focality of the applied stimulation could have played an important role in the results. Moreover, our participants were university students with a high percentage of females, and they were all young participants. However, approach and avoidance brain processing could be affected by developmental changes or modulated by gender. Thus, future studies should also include adult and more male participants.

\section{Conclusions}

Anodal stimulation on rSTS had no effect on reading approach content; however, it did have a general effect on reading social content about persons. Plausibly, the positive effect of anodal stimulation on memorization of approach occurs after reading, during the process of encoding the meaning of the sentences. BIS and BAS affective traits modulate tDCS effect on reading speed, which benefits low BAS and BIS participants, plausibly due to problems with allocation of additional resources in high BAS and BIS participants. Likewise, low-BIS participants seem to show a motivational bias towards paying less attention to avoidance content. In short, anodal tDCS appears to improve reading speed of social content, but not specifically of interpersonal or approach content. The modulation of affective traits in modulating tDCS effects has emerged as a relevant factor. More attention to this modulation would be necessary in future research on cognitive improvement by tDCS stimulation. Our results are relevant for brain research on the mentalizing network to understand social content in sentences and its relationship with differences in personality traits.

Author Contributions: Conceptualization and design, H.M., C.R. and I.P.; interpretation of data, H.M., C.R. and S.N.Y.; data collection, C.R. and S.N.Y.; drafting the article C.R. and H.M.; critically revising the article, H.M. and C.R.; analysis of data, H.M., C.R. and S.N.Y.; tDCS protocol implementation, I.P. and C.R. All authors have read and agreed to the published version of the manuscript.

Funding: This study was supported by the Spanish Ministry of Economy, Industry and Competitiveness (Grant PSI2017-84527-P).

Institutional Review Board Statement: The study was conducted according to the guidelines of the Declaration of Helsinki, and approved by the Ethics Committee) of University of La Laguna (NAME OF INSTITUTE (protocol code CEIBA 2017-0272), 22 December 2017).

Informed Consent Statement: Informed consent was obtained from all subjects involved in the study.

Data Availability Statement: The data presented in this study are openly available in Figshare at 10.6084/m9.figshare.16867144.

Acknowledgments: We thank the Canarian Agency of Research, Innovation and Knowledge Society (NEUROCOG project) and The Colegio Oficial de la Psicología de Las Palmas, and the European Regional Development Funds.

Conflicts of Interest: The authors declare no conflict of interest.

\section{References}

1. Dodell-Feder, D.; Koster-Hale, J.; Bedny, M.; Saxe, R. FMRI Item Analysis in a Theory of Mind Task. NeuroImage 2011, 55, 705-712. [CrossRef] [PubMed]

2. Kennedy, D.P.; Adolphs, R. The Social Brain in Psychiatric and Neurological Disorders. Trends Cogn. Sci. $2012,16,559-572$. [CrossRef] [PubMed]

3. Spunt, R.P.; Falk, E.B.; Lieberman, M.D. Dissociable Neural Systems Support Retrieval of How and Why Action Knowledge. Psychol. Sci. 2010, 21, 1593-1598. [CrossRef] [PubMed]

4. Gobbini, M.I.; Koralek, A.C.; Bryan, R.E.; Montgomery, K.J.; Haxby, J.V. Two Takes on the Social Brain: A Comparison of Theory of Mind Tasks. J. Cogn. Neurosci. 2007, 19, 1803-1814. [CrossRef]

5. Garofalo, S.; Timmermann, C.; Battaglia, S.; Maier, M.E.; di Pellegrino, G. Mediofrontal Negativity Signals Unexpected Timing of Salient Outcomes. J. Cogn. Neurosci. 2017, 29, 718-727. [CrossRef] [PubMed] 
6. Battaglia, S.; Garofalo, S.; di Pellegrino, G.; Starita, F. Revaluing the Role of VmPFC in the Acquisition of Pavlovian Threat Conditioning in Humans. J. Neurosci. 2020, 40, 8491-8500. [CrossRef] [PubMed]

7. Battaglia, S.; Serio, G.; Scarpazza, C.; D'Ausilio, A.; Borgomaneri, S. Frozen in (e)Motion: How Reactive Motor Inhibition Is Influenced by the Emotional Content of Stimuli in Healthy and Psychiatric Populations. Behav. Res. Ther. 2021, $146,103963$. [CrossRef] [PubMed]

8. Watson, R.; Latinus, M.; Charest, I.; Crabbe, F.; Belin, P. People-Selectivity, Audiovisual Integration and Heteromodality in the Superior Temporal Sulcus. Cortex 2014, 50, 125-136. [CrossRef] [PubMed]

9. Wong, C.; Gallate, J. The function of the anterior temporal lobe: A review of the empirical evidence. Brain Res. 2012, 1449, 94-116. [CrossRef] [PubMed]

10. Ellena, G.; Starita, F.; Haggard, P.; Làdavas, E. The Spatial Logic of Fear. Cognition 2020, 203, 104336. [CrossRef]

11. Candini, M.; Battaglia, S.; Benassi, M.; di Pellegrino, G.; Frassinetti, F. The Physiological Correlates of Interpersonal Space. Sci. Rep. 2021, 11, 2611. [CrossRef]

12. Johnson, M.H.; Senju, A.; Tomalski, P. The Two-Process Theory of Face Processing: Modifications Based on Two Decades of Data from Infants and Adults. Neurosci. Biobehav. Rev. 2015, 50, 169-179. [CrossRef] [PubMed]

13. Pelphrey, K.A.; Morris, J.P. Brain Mechanisms for Interpreting the Actions of Others from Biological-Motion Cues. Curr. Dir. Psychol. Sci. 2006, 15, 136-140. [CrossRef]

14. Pelphrey, K.A.; Carter, E.J. Brain Mechanisms for Social Perception. Ann. N. Y. Acad. Sci. 2008, 1145, 283-299. [CrossRef] [PubMed]

15. Saitovitch, A.; Bargiacchi, A.; Chabane, N.; Brunelle, F.; Samson, Y.; Boddaert, N.; Zilbovicius, M. Social Cognition and the Superior Temporal Sulcus: Implications in Autism. Rev. Neurol. (Paris) 2012, 168, 762-770. [CrossRef]

16. Yang, D.Y.J.; Rosenblau, G.; Keifer, C.; Pelphrey, K.A. An Integrative Neural Model of Social Perception, Action Observation, and Theory of Mind. Neurosci. Biobehav. Rev. 2015, 51, 263-275. [CrossRef]

17. Flores, L.E.; Eckstrand, K.L.; Silk, J.S.; Allen, N.B.; Ambrosia, M.; Healey, K.L.; Forbes, E.E. Adolescents' Neural Response to Social Reward and Real-World Emotional Closeness and Positive Affect. Cogn. Affect. Behav. Neurosci. 2018, 18, 705-717. [CrossRef] [PubMed]

18. Pelphrey, K.A.; Viola, R.J.; McCarthy, G. When Strangers Pass: Processing of Mutual and Averted Social Gaze in the Superior Temporal Sulcus. Psychol. Sci. 2004, 15, 598-603. [CrossRef] [PubMed]

19. Ross, L.A.; Olson, I.R. Social Cognition and the Anterior Temporal Lobes. NeuroImage 2010, 49, 3452-3462. [CrossRef] [PubMed]

20. Tavares, P.; Lawrence, A.D.; Barnard, P.J. Paying Attention to Social Meaning: An FMRI Study. Cereb. Cortex 2008, 18, 1876-1885. [CrossRef]

21. Marrero, H.; Urrutia, M.; Beltrán, D.; Gámez, E.; Díaz, J.M. Understanding Approach and Avoidance in Verbal Descriptions of Everyday Actions: An ERP Study. Cogn. Affect. Behav. Neurosci. 2017, 17, 612-624. [CrossRef] [PubMed]

22. Marrero, H.; Gámez, E.; Diaz, J.M.; Urrutia, M.; de Vega, M. Carefully encoding approach and avoidance body locomotion with interpersonal conduct in narrated interactions. Can. J. Exp. Psychol. 2015, 69, 190-199. [CrossRef] [PubMed]

23. Yavari, F.; Jamil, A.; Mosayebi Samani, M.; Vidor, L.P.; Nitsche, M.A. Basic and Functional Effects of Transcranial Electrical Stimulation (TES)—An Introduction. Neurosci. Biobehav. Rev. 2018, 85, 81-92. [CrossRef] [PubMed]

24. Barker, A.T.; Jalinous, R.; Freeston, I.L. Non-invasive magnetic stimulation of human motor cortex. Lancet 1985, 325, 1106-1107. [CrossRef]

25. George, M.S.; Aston-Jones, G. Noninvasive Techniques for Probing Neurocircuitry and Treating Illness: Vagus Nerve Stimulation (VNS), Transcranial Magnetic Stimulation (TMS) and Transcranial Direct Current Stimulation (TDCS). Neuropsychopharmacology 2010, 35, 301-316. [CrossRef] [PubMed]

26. Nitsche, M.A.; Cohen, L.G.; Wassermann, E.M.; Priori, A.; Lang, N.; Antal, A.; Paulus, W.; Hummel, F.; Boggio, P.S.; Fregni, F.; et al. Transcranial Direct Current Stimulation: State of the Art 2008. Brain Stimulat. 2008, 1, 206-223. [CrossRef] [PubMed]

27. Borgomaneri, S.; Battaglia, S.; Garofalo, S.; Tortora, F.; Avenanti, A.; di Pellegrino, G. State-Dependent TMS over Prefrontal Cortex Disrupts Fear-Memory Reconsolidation and Prevents the Return of Fear. Curr. Biol. 2020, 30, 3672-3679.e4. [CrossRef]

28. Brunelin, J.; Mondino, M.; Gassab, L.; Haesebaert, F.; Gaha, L.; Suaud-Chagny, M.-F.; Saoud, M.; Mechri, A.; Poulet, E. Examining Transcranial Direct-Current Stimulation (TDCS) as a Treatment for Hallucinations in Schizophrenia. Am. J. Psychiatry 2012, 169, 719-724. [CrossRef] [PubMed]

29. Kuo, M.-F.; Paulus, W.; Nitsche, M.A. Therapeutic Effects of Non-Invasive Brain Stimulation with Direct Currents (TDCS) in Neuropsychiatric Diseases. NeuroImage 2014, 85, 948-960. [CrossRef]

30. Loo, C.K.; Alonzo, A.; Martin, D.; Mitchell, P.B.; Galvez, V.; Sachdev, P. Transcranial Direct Current Stimulation for Depression: 3-Week, Randomised, Sham-Controlled Trial. Br. J. Psychiatry 2012, 200, 52-59. [CrossRef] [PubMed]

31. Marrero, H.; Yagual, S.N.; García-Marco, E.; Gámez, E.; Beltrán, D.; Díaz, J.M.; Urrutia, M. Enhancing Memory for Relationship Actions by Transcranial Direct Current Stimulation of the Superior Temporal Sulcus. Brain Sci. 2020, 10, 497. [CrossRef] [PubMed]

32. Cancer, A.; Antonietti, A. TDCS Modulatory Effect on Reading Processes: A Review of Studies on Typical Readers and Individuals with Dyslexia. Front. Behav. Neurosci. 2018, 12, 1-12. [CrossRef] [PubMed]

33. Thomson, J.M.; Doruk, D.; Mascio, B.; Fregni, F.; Cerruti, C. Transcranial Direct Current Stimulation Modulates Efficiency of Reading Processes. Front. Hum. Neurosci. 2015, 9, 1-9. [CrossRef] 
34. Barsalou, L.W. Simulation, Situated Conceptualization, and Prediction. Philos. Trans. R. Soc. B Biol. Sci. 2009, 364, 1281-1289. [CrossRef]

35. Zwaan, R.A. The immersed experiencer: Toward an embodied theory of language comprehension. In The Psychology of Learning and Motivation; Ross, B.H., Ed.; Academic Press: New York, NY, USA, 2004; Volume 44, pp. 35-62.

36. Gray, J.A.A. A critique of Eysenck's theory of personality. In A Model for Personality; Eysenck, H.J., Ed.; Springer: Berlin/Heidelberg, Germany, 1981; pp. 246-276.

37. Metuki, N.; Sela, T.; Lavidor, M. Enhancing Cognitive Control Components of Insight Problems Solving by Anodal TDCS of the Left Dorsolateral Prefrontal Cortex. Brain Stimulat. 2012, 5, 110-115. [CrossRef] [PubMed]

38. Blumenthal, T.D. Extraversion, Attention, and Startle Response Reactivity. Personal. Individ. Differ. 2001, 31, 495-503. [CrossRef]

39. Sela, T.; Ivry, R.B.; Lavidor, M. Prefrontal Control during a Semantic Decision Task That Involves Idiom Comprehension: A Transcranial Direct Current Stimulation Study. Neuropsychologia 2012, 50, 2271-2280. [CrossRef] [PubMed]

40. Giakoumaki, S.G.; Roussos, P.; Tsapakis, E.M.; Koiliari, E.; Pasparakis, E.; Zouraraki, C.; Bitsios, P. Cognitive and Personality Analysis of Startle Reactivity in a Large Cohort of Healthy Males. Biol. Psychol. 2013, 94, 582-591. [CrossRef]

41. LaRowe, S.D.; Patrick, C.J.; Curtin, J.J.; Kline, J.P. Personality Correlates of Startle Habituation. Biol. Psychol. 2006, 72, 257-264. [CrossRef] [PubMed]

42. Eysenck, M.W.; Derakshan, N.; Santos, R.; Calvo, M.G. Anxiety and cognitive performance: Attentional control theory. Emotion 2007, 7, 336-353. [CrossRef] [PubMed]

43. Faul, F.; Erdfelder, E.; Lang, A.G.; Buchner, A. G*Power 3: A Flexible Statistical Power Analysis Program for the Social, Behavioral, and Biomedical Sciences. Behav. Res. Methods 2007, 39, 175-191. [CrossRef] [PubMed]

44. Oldfield, R.C. The assessment and analysis of handedness: The Edinburgh inventory. Neuropsychologia 1971, 9, 97-113. [CrossRef]

45. Carver, C.S.; White, T.L. Behavioral Inhibition, Behavioral Activation, and Affective Responses to Impending Reward and Punishment: The BIS/BAS Scales. J. Pers. Soc. Psychol. 1994, 67, 319-333. [CrossRef]

46. Nitsche, M.A.; Doemkes, S.; Karaköse, T.; Antal, A.; Liebetanz, D.; Lang, N.; Tergau, F.; Paulus, W. Shaping the Effects of Transcranial Direct Current Stimulation of the Human Motor Cortex. J. Neurophysiol. 2007, 97, 3109-3117. [CrossRef]

47. Huang, Y.; Datta, A.; Bikson, M.; Parra, L.C. Realistic Volumetric-Approach to Simulate Transcranial Electric StimulationROAST-A Fully Automated Open-Source Pipeline. J. Neural Eng. 2019, 16, 056006. [CrossRef]

48. Díez, E.; Gómez-Ariza, C.J.; Díez-Álamo, A.M.; Alonso, M.A.; Fernandez, A. The Processing of Semantic Relatedness in the Brain: Evidence from Associative and Categorical False Recognition Effects Following Transcranial Direct Current Stimulation of the Left Anterior Temporal Lobe. Cortex 2017, 93, 133-145. [CrossRef] [PubMed]

49. Zwissler, B.; Sperber, C.; Aigeldinger, S.; Schindler, S.; Kissler, J.; Plewnia, C. Shaping Memory Accuracy by Left Prefrontal Transcranial Direct Current Stimulation. J. Neurosci. 2014, 34, 4022-4026. [CrossRef]

50. Paulus, W. Transcranial electrical stimulation (tES-tDCS; tRNS, tACS) methods. Neuropsychol. Rehabil. 2011, $21,602-617$. [CrossRef] [PubMed]

51. Brunoni, A.R.; Amadera, J.; Berbel, B.; Volz, M.S.; Rizzerio, B.G.; Fregni, F. A Systematic Review on Reporting and Assessment of Adverse Effects Associated with Transcranial Direct Current Stimulation. Int. J. Neuropsychopharmacol. 2011, 14, 1133-1145. [CrossRef] [PubMed]

52. Kauschke, C.; Bahn, D.; Vesker, M.; Schwarzer, G. The Role of Emotional Valence for the Processing of Facial and Verbal Stimuli-Positivity or Negativity Bias? Front. Psychol. 2019, 10, 1654. [CrossRef] [PubMed]

53. Kaup, B.; Lüdtke, J.; Zwaan, R.A. Processing Negated Sentences with Contradictory Predicates: Is a Door That Is Not Open Mentally Closed? J. Pragmat. 2006, 38, 1033-1050. [CrossRef] 